\title{
Stamps for nanoimprint lithography by extreme ultraviolet interference lithography
}

\author{
Sunggook Park, Helmut Schift, ${ }^{\text {a) }}$ Harun H. Solak, and Jens Gobrecht \\ Laboratory for Micro- and Nanotechnology, Paul Scherrer Institut, 5232 Villigen PSI, Switzerland
}

(Received 2 June 2004; accepted 13 October 2004; published 10 December 2004)

\begin{abstract}
Extreme ultraviolet interference lithography (EUV-IL) was employed to fabricate large area stamps with patterns of sub-50 $\mathrm{nm}$ half pitch for nanoimprint lithography (NIL). The resist patterns exposed by EUV-IL were transferred into underlying $\mathrm{Si}$ or $\mathrm{SiO}_{2} / \mathrm{Si}$ substrates by reactive ion etching. With this method, NIL stamps with feature sizes as small as $25 \mathrm{~nm}$ and patterned areas as large as 1 $\times 1 \mathrm{~mm}^{2}$ were successfully produced. Using those stamps in NIL, the patterns with aspect ratio up to 2 were replicated with good replication fidelity. Increasing aspect ratio for the stamp patterns first results in distortion and discontinuity and then, the ripping of partial structures in the imprinted patterns. () 2004 American Vacuum Society. [DOI: 10.1116/1.1828087]
\end{abstract}

\section{INTRODUCTION}

Nanoimprint lithography (NIL) is an emerging patterning technology based on the molding of resists. ${ }^{1-4}$ As in any other molding based technology, one of the most important requirements for NIL is to obtain reliable stamps with desired structures which can be repeatedly used without damage during demolding. Most stamps with nanostructures below $120 \mathrm{~nm}$ in feature size have been fabricated via electron beam writing in a resist, followed by Si reactive ion etching (RIE). With this method, single lines, small arrays of dots, or sparsely distributed structures as small as $10 \mathrm{~nm}$ can be made. For high density and large area patterns of such feature sizes, however, the use of electron beam lithography is hampered because the proximity effect of the electron beam contributes to the pattern formation significantly and, thus, there are still needs for the technology which enables reliable fabrication of NIL stamps with high density patterns at the sub-50 $\mathrm{nm}$ scale.

Extreme ultraviolet interference lithography (EUV-IL) is a suitable candidate for the fabrication of NIL stamps with high density features of sub-50 $\mathrm{nm}$ half pitches. ${ }^{5,6}$ In EUVIL, the first order diffracted EUV beams from the diffraction gratings interfere in the center to form one- or twodimensional periodic patterns such as gratings and dot arrays. ${ }^{6}$ The half pitch of the EUV exposed patterns is reduced by a factor of 2 and $\sqrt{2}$ from that of the linear diffraction gratings in the two- and four-beam interference, respectively. The half pitch reduction of large area patterns can also be achieved by photolithography using a projection printer. ${ }^{7}$ However, by using EUV one can reach much higher lateral resolution, as demonstrated in the patterning of linear gratings with $19 \mathrm{~nm}$ half pitch. ${ }^{5,6}$ As the feature size decreases, the transfer of the pattern into underlying $\mathrm{Si}$ or $\mathrm{SiO}_{2}$ substrates with desired aspect ratio becomes more difficult to achieve, which is needed to implement EUV-IL as a reliable means of fabricating NIL stamps. This can be made possible

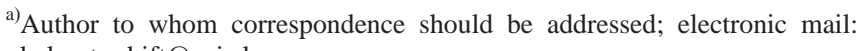
helmut.schift@psi.ch only when the production of EUV exposed patterns with reasonable aspect ratio are combined with a proper choice of process technology and optimization of each process which includes EUV exposure, development, etching pattern transfer.

In this contribution, we will present the fabrication of NIL stamps via EUV-IL and the first imprint results using those stamps. Periodic patterns of gratings and dots with half pitches down to $25 \mathrm{~nm}$ were successfully transferred into underlying $\mathrm{Si}$ or $\mathrm{SiO}_{2} / \mathrm{Si}$ substrates, demonstrating the feasibility of EUV-IL in the production of NIL stamps. Imprinting of those stamps into a resist film shows good replication fidelity for patterns of aspect ratio up to 2 .

\section{EXPERIMENT}

For the fabrication of NIL stamps via EUV-IL, two strategies were used (Fig. 1); the transfer of EUV exposed patterns in poly(methylmethacrylate) (PMMA, $M_{w}$ $=600 \mathrm{~kg} / \mathrm{mol}$, thickness $=45-100 \mathrm{~nm}$ ) either into Si wafers or into thermally grown, 200-nm-thick $\mathrm{SiO}_{2}$ layer. The exposure of EUV beams with wavelengths of $13.4 \mathrm{~nm}$ was performed in the XIL beam line at Swiss Light Source (SLS) at PSI. ${ }^{6}$ Then, the exposed samples were developed in 1:3 MIBK:IPA solution for 25-30 s. For the pattern transfer into Si substrates [Fig. 1(a)], the PMMA patterns were first transferred into an intermediate $\mathrm{Cr}$ layer of $20 \mathrm{~nm}$ in thickness by reactive ion etching (RIE) using a gas mixture of $\mathrm{Cl}_{2}: \mathrm{CO}_{2}$ $=100: 100 \mathrm{sccm}$ at $100 \mathrm{~W}$. This was followed by Si RIE (Oxford Plasmafab 100) using the Cr layer as a hard etch mask, which was performed using a gas mixture of $\mathrm{SF}_{6}: \mathrm{CHF}_{3}: \mathrm{O}_{2}=2: 40: 5 \mathrm{sccm}$ at a bias and power of $700 \mathrm{~V}$ and $200 \mathrm{~W}$, respectively. This leads to an almost vertical sidewall profile with an etching rate of $60 \mathrm{~nm} / \mathrm{min}$. Finally, the residual $\mathrm{Cr}$ layer was stripped with a wet chemical method to obtain final $\mathrm{Si}$ stamps. For $\mathrm{SiO}_{2}$ stamps, on the other hand, the EUV exposed PMMA patterns were directly used as an etch mask [Fig. 1(b)]. For the $\mathrm{SiO}_{2} \mathrm{RIE}$ a gas mixture of $\mathrm{CHF}_{3}: \mathrm{O}_{2}=50: 1 \mathrm{sccm}$ was used. The surfaces for both types of stamps were coated with a fluorinated silane layer in the gas phase to reduce sticking between resists and 


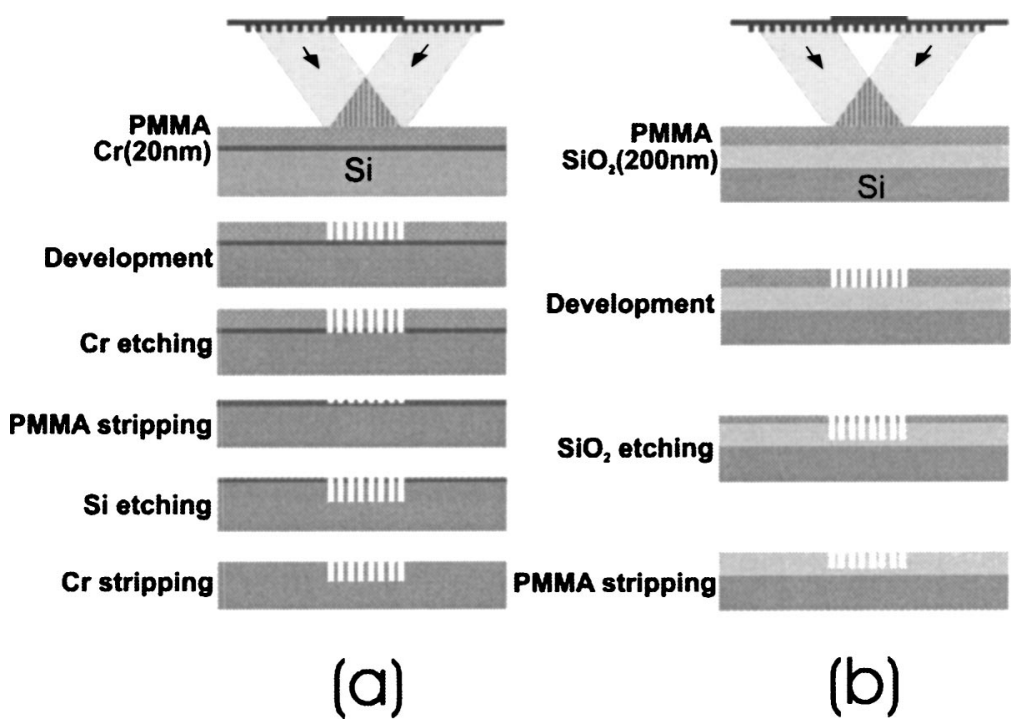

FIG. 1. Process schemes of transferring resist patterns exposed by EUV-IL into (a) $\mathrm{Si}$ and (b) $\mathrm{SiO}_{2} / \mathrm{Si}$ substrates to be used as NIL stamps.

stamps during demolding. ${ }^{8,9}$ It should be mentioned that for such small features the anti-adhesive coating has to be performed in the gas phase to prevent issues related to capillary into the small sized patterns.

As substrates for imprints, thin films $(\sim 330 \mathrm{~nm})$ of PMMA $\left(M_{w}=25 \mathrm{~kg} / \mathrm{mol}\right)$ were spin coated on Si substrates, followed by baking on a hot plate for $1 \mathrm{~min}$ at $170{ }^{\circ} \mathrm{C}$. The imprinting was performed at $180{ }^{\circ} \mathrm{C}$ and 50 bars for $10 \mathrm{~min}$ and the demolding was done at $70^{\circ} \mathrm{C}$.

The patterns were characterized by scanning electron microscopy (SEM, LEICA e-beam pattern generator) and atomic force microscopy (AFM, Digital Instruments Nanoscope III/Dimension 3100). AFM measurements were performed in tapping mode using a $\mathrm{Si}$ cantilever with a tip radius of $\sim 15 \mathrm{~nm}$ (Nanoworld-NCHR, $330 \mathrm{kHz}$ ).

\section{RESULTS AND DISCUSSION}

Most of the diffraction gratings were written with electron beam lithography except for that of $25 \mathrm{~nm}$ half pitch which was produced by EUV-IL using its half pitch reducing capability. The patterned area by EUV-IL is determined by several factors, such as, the diameter of synchrotron beam, the size of diffraction gratings, the size of pinholes used to set the coherently illuminated area, and the distance between the diffraction grating and the substrate. The largest area achieved in this experiment is $1 \times 1 \mathrm{~mm}^{2}$. However, by going to smaller sizes for the pinholes, we expect to be able to coherently illuminate the diffraction gratings on the area as large as $5 \times 5 \mathrm{~mm}^{2}$.

For reliable pattern transfer into underlying substrates, it is desirable to have patterns with highest possible aspect ratio for EUV exposed samples which do not collapse during development and are stable enough to withstand the postetching process. For that the optimal initial PMMA thickness has to be determined first. Figure 2 shows AFM images of EUV exposed PMMA after development, where an initial PMMA thickness of 105 and $45 \mathrm{~nm}$ was used for patterns with 50 and $25 \mathrm{~nm}$ half pitches, respectively. By carefully controlling over the exposure doses and development conditions, patterns with aspect ratio of 2 could be achieved for PMMA, as seen in the figure for patterns of $50 \mathrm{~nm}$ half pitch. For the linear gratings of $25 \mathrm{~nm}$ half pitch the AFM tip could not probe the structure depth. However, further pattern transfer indicates the depth close to $35 \mathrm{~nm}$.

The EUV exposed patterns were then transferred into the substrates to be used as NIL stamps. Figure 2 shows SEM images for Si stamps with different half pitches produced by using a 20-nm-thick $\mathrm{Cr}$ etch mask, as described in Fig. 1(a). The depth of patterns was $150 \mathrm{~nm}$. The method is suitable to produce high aspect ratio patterns due to high etch selectivity of Si relative to $\mathrm{Cr}$ and has already demonstrated the production of NIL stamps with an array of pillars of $200 \mathrm{~nm}$ half pitch and aspect ratio as high as 4 for polymer photonic band gap devices. ${ }^{10,11}$ However, significant defects such as thinning and discontinuity of the lines begin to occur as feature sizes become smaller than $35.5 \mathrm{~nm}$ half pitch, despite the use of a thin Cr thickness which in general helps reduce a critical

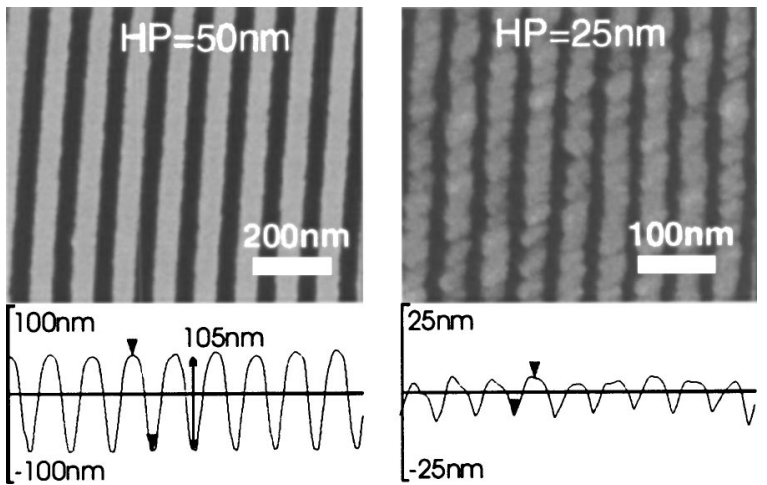

FIG. 2. AFM images of EUV exposed PMMA patterns. The initial PMMA thickness used for exposure was 105 and $45 \mathrm{~nm}$ for patterns of 50 and $25 \mathrm{~nm}$ half pitch, respectively. Half pitch is denoted as HP in figures. 

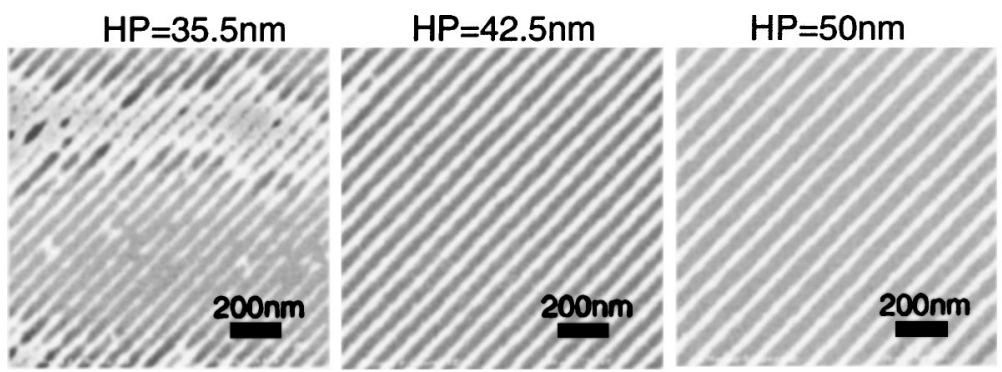

(a)
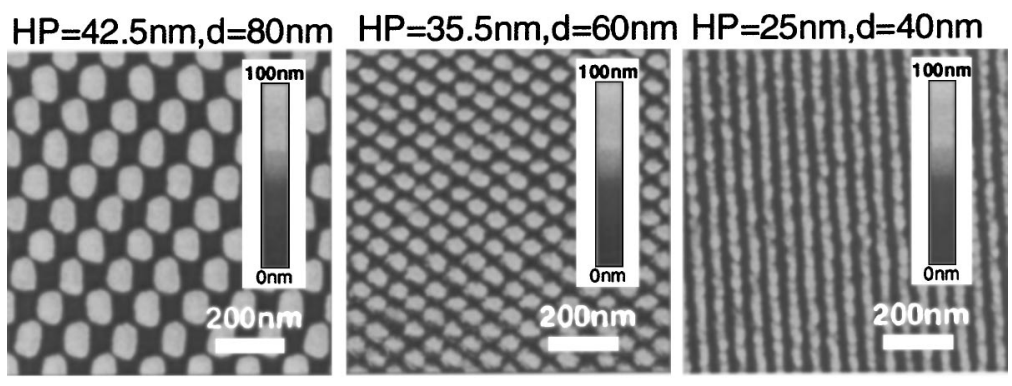

(b)

FIG. 3. (a) SEM images for Si stamps with lines of 35.5, 42.5, and $50 \mathrm{~nm}$ half pitches which were produced using the procedure shown in Fig. 1(a). The depth of all patterns was $150 \mathrm{~nm}$, as measured from AFM. (b) AFM images for $\mathrm{SiO}_{2}$ stamps produced using the procedure shown in Fig. 1(b).

dimension loss during etching of extremely small features. ${ }^{12,13}$ This is partially due to slightly isotropic characteristics in our $\mathrm{Cr}$ etching process, but it also seems to be the reason that the EUV exposed patterns are not stable enough to sustain the RIE process, which might be related to the efficiency of the diffraction gratings and the development condition. In addition, the slight intensity oscillation of EUV light intensity occurring near the edge of the patterned area due to Fresnel diffraction makes it more difficult to achieve homogeneous pattern transfer for extremely small features. As a means of solving this problem, the pattern could be exposed again to an electron beam to cross-link the PMMA, increasing the lateral etch resistance during $\mathrm{Cr}$ etching. ${ }^{6}$ However, this is not practical for large area patterns.

Having seen the limitations of the process using a $\mathrm{Cr}$ hard etch mask, we applied another strategy shown in Fig. 1(b), which utilizes the PMMA pattern as an etch mask to directly etch into $\mathrm{SiO}_{2}$. Examples of the NIL stamps made in this method are shown in Fig. 3(b). All the exposed patterns were well transferred into the $\mathrm{SiO}_{2} / \mathrm{Si}$ substrate over the patterned areas of $80 \times 80 \mu \mathrm{m}^{2}$ for dots and $1000 \times 150 \mu \mathrm{m}^{2}$ for lines without any apparent limitation. However, because the etch selectivity of $\mathrm{SiO}_{2}$ with respect to PMMA under the RIE conditions used is slightly higher than 1 , only aspect ratio close to that of the EUV exposed PMMA patterns could be achieved for the patterns transferred into $\mathrm{SiO}_{2}$. The highest aspect ratio achieved by this process was 1.6 and, however, this could be further improved by using a resist with increased etching resistance. Nevertheless, the results on the pattern transfer clearly demonstrate the feasibility of EUV-IL to produce large area, high density NIL stamps in the sub$50 \mathrm{~nm}$ feature sizes, when combined with an appropriate pattern transfer process.

Using the stamps fabricated via EUV-IL, imprints were performed on PMMA coated Si substrates. Figure 4 presents imprinted PMMA patterns for dots and lines of 35.5 and $25 \mathrm{~nm}$ half pitches, respectively. When compared with the stamps shown in Fig. 3(b), slight difference in the lateral dimension is seen. Apart from the influence from the AFM tip radius, this might be due to the relaxation of PMMA after demolding. In general, however, it shows good replication fidelity, which demonstrates the capability of NIL in the replication of high density and large area features in the sub$50 \mathrm{~nm}$ half pitch regime.

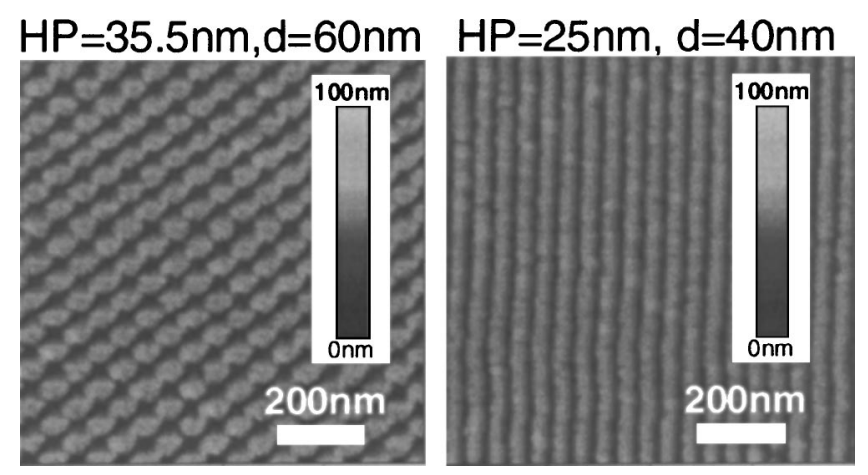

FIG. 4. AFM images for imprinted PMMA with $\mathrm{SiO}_{2}$ stamps of pillars and gratings with 25.5 and $25 \mathrm{~nm}$ half pitches, respectively. 


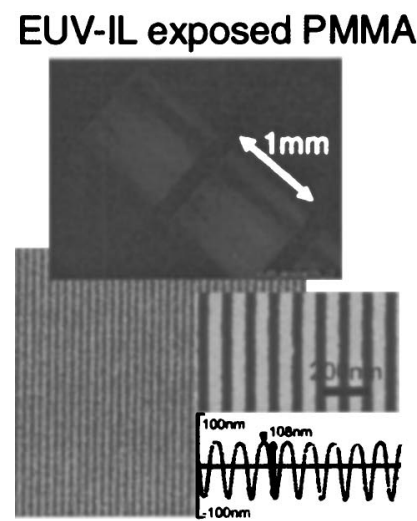

(a)

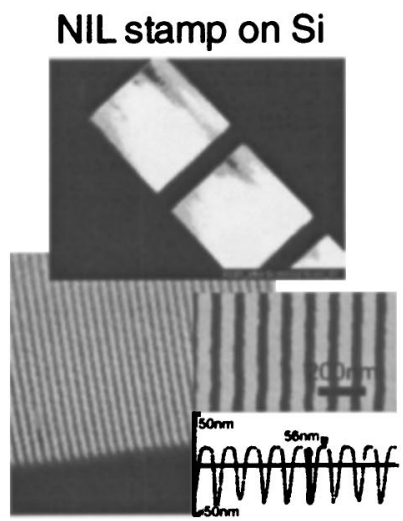

(b)

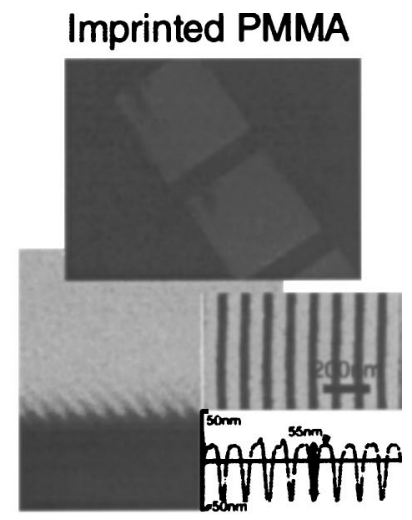

(c)

FIG. 5. Dark field optical microscope, SEM, and AFM images: (a) for EUV-IL exposed PMMA, (b) after transferring the pattern into Si to be used as a NIL stamp, and (c) imprinted PMMA using the stamp shown in (b). The half pitch of the pattern is $50 \mathrm{~nm}$ and the area of patterns is $1 \times 1 \mathrm{~mm}^{2}$.

Figure 5 shows the EUV exposed PMMA, the corresponding NIL stamp, and imprinted PMMA for the largest area pattern that we have produced by now. The patterned area is $1 \times 1 \mathrm{~mm}^{2}$, the half pitch of the linear gratings is $50 \mathrm{~nm}$, and the aspect ratio is 1 . The brightness shown in the dark field microscope images at the $45^{\circ}$ rotation relative to the gratings indicates the formation of one-dimensional periodic patterns. This again proves the successful pattern transfer and imprinting with good replication fidelity over the whole patterned areas.

Then, using the largest area diffraction grating we fabricated Si stamps with different aspect ratios in order to investigate the ability of NIL for high aspect ratio patterns. No failure was observed upon repeated imprints for the aspect ratio below 2. However, as the aspect ratio exceeds 2, it was difficult to achieve good replication fidelity. Figure 6 shows imprinted PMMA patterns with the stamp of aspect ratio 2. A few distortions and discontinuity of the gratings appeared over the patterned area, which was not present in the corresponding stamp. This can be attributed to the increased friction between the stamp and PMMA during demolding, and the increased stress between the neighboring lines associated

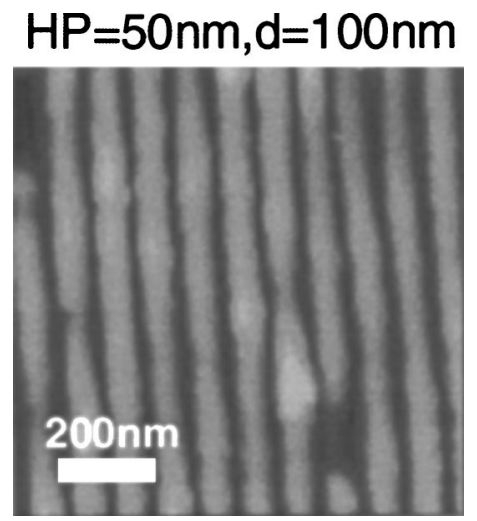

FIG. 6. AFM image for the imprinted PMMA of $50 \mathrm{~nm}$ half pitch and $100 \mathrm{~nm}$ depth patterned over the area of $1 \times 1 \mathrm{~mm}^{2}$. with the increased density of the patterns. ${ }^{14,15}$ Further increase in the aspect ratio results in catastrophic failure during imprint, namely the partial ripping of the resist.

\section{CONCLUSION}

We have shown a fabrication process for NIL stamps with patterns of high density and large area at the sub-50 nm half pitch scale via EUV-IL. While the pattern transfer process using a $\mathrm{Cr}$ hard etch mask is suitable for producing Si stamps with high aspect ratio patterns, for extreme small resist patterns the direct use of the PMMA mask on $\mathrm{SiO}_{2} / \mathrm{Si}$ was found to be a better choice. However, the fabrication of high aspect ratio stamps is limited. By choosing an appropriate pattern transfer process, we could produce the stamp with feature size as small as $25 \mathrm{~nm}$ and aspect ratio close to 2, indicating the feasibility of EUV-IL in the NIL stamps fabrication. Once such stamps are fabricated, then the patterns can further be replicated using NIL. We were able to imprint those stamps with good replication fidelity for aspect ratio up to 2. However, such limit can further be overcome by improving the fabrication process, for example, specific sidewall profiles, reduced surface roughness in NIL stamps, and improving anti-adhesion properties in the stamp surfaces. It might also help to find new resist polymers and demolding strategies such as demolding at close to the glass transition temperature in order to reduce stress and tendency of breaking structures.

\section{ACKNOWLEDGMENTS}

The authors would like to thank Konrad Vogelsang (Paul Scherrer Institut) for the technical support. All EUV-IL exposures were made at the XIL beamline of the Swiss Light Source (SLS) at PSI. This work was partially funded by EC through project NaPa (Contract No. NMP4-CT-2003500120).

${ }^{1}$ S. Y. Chou and P. R. Krauss, Microelectron. Eng. 35, 237 (1997).

${ }^{2}$ H. Schift and L. J. Heyderman, in Alternative Lithography, edited by D. J. 
Lockwood (Kluwer/Plenum, New York, 2003), p. 46.

${ }^{3}$ S. Zankovych, T. Hoffmann, J. Seekamp, J.-U. Bruch, and C. M. Sotomayor Torres, Nanotechnology 12, 91 (2001).

${ }^{4}$ C. M. Sotomayor Torres, S. Zankovych, J. Seekamp, A. P. Kam, C. Clavijo Cedeno, T. Hoffmann, J. Ahopelto, F. Reuther, K. Pfeiffer, G. Bleidiessel, G. Gruetzner, M. V. Maximov, and B. Heidari, Mater. Sci. Eng. 23, 23 (2003).

${ }^{5}$ H. H. Solak, D. He, W. Li, S. Singh-Gasson, F. Cerrina, B. H. Sohn, X. M. Yang, and P. Nealey, Appl. Phys. Lett. 75, 2328 (1999).

${ }^{6}$ H. H. Solak, C. David, J. Gobrecht, V. Golovkina, F. Cerrina, S. O. Kim, and P. F. Nealey, Microelectron. Eng. 67-68, 56 (2003).

${ }^{7}$ M. Burkhardt, A. Yen, C. Progler, and G. Wells, Microelectron. Eng. 41-42, 91 (1998).

${ }^{8}$ S. Park, C. Padeste, H. Schift, and J. Gobrecht, Microelectron. Eng. 6768, 252 (2003).
${ }^{9}$ S. Park, H. Schift, C. Padeste, B. Schnyder, and J. Gobrecht, Proceedings of the Materials Research Society'03 Fall Meeting, Boston, MA, 1-5 December 2003, Vol. EXS-2, p. 37.

${ }^{10}$ C.-S. Kee, S.-P. Han, K. B. Yoon, C.-G. Choi, H. K. Sung, M. Y. Jeong, S. S. Oh, H. Y. Park, S. Park, and H. Schift, Appl. Phys. Lett. (submitted).

${ }^{11}$ H. Schift, S. Park, C.-G. Choi, C.-S. Kee, S.-P. Han, K.-B. Yoon, and J. Gobrecht, accepted conference contribution to TNT 2004 conference.

${ }^{12}$ A. Y. Abdo, L. Zheng, A. Wei, A. Mikkelson, G. Nellis, R. L. Engelstand, and E. G. Lovell, Microelectron. Eng. 73-74, 161 (2004).

${ }^{13}$ D. Resnick, W. Dauksher, D. Mancini, K. Nordquist, E. Ainley, K. Gehoski, J. Baker, T. Bailey, B. Choi, S. Johnson, S. Sreenivasan, J. Ekerdt, and C. Wilson, Proc. SPIE 4688, 205 (2002).

${ }^{14}$ H. Schift, L. J. Heyderman, C. Padeste, and J. Gobrecht, Microelectron. Eng. 61-62, 423 (2002).

${ }^{15}$ H. Schift, L. J. Heyderman, and J. Gobrecht, Chimia 56, 543 (2002). 